\title{
Evolución de la docencia en Teoría de Grafos en las últimas décadas
}

\section{Jordán ${ }^{\mathrm{a}}$, E. Sanabria ${ }^{\mathrm{b}}$, J. R. Torregrosa $a^{\mathrm{a}}$, A. Cordero ${ }^{\mathrm{a}}$ y A. Conejero ${ }^{\mathrm{c}}$}

Instituto Universitario de Matemática Multidisciplinar, Universitat Politècnica de València, ${ }^{b}$ Departamento de Matemática Aplicada, Universitat Politècnica de València y

${ }^{c}$ Instituto Universitario de Matemática Pura y Aplicada, Universitat Politècnica de València, (cjordan@mat.upv.es, esanabri@mat.upv.es, jrtorre@mat.upv.es, acordero@mat.upv.es, aconejero@mat.upv.es)

\begin{abstract}
In this work we present the innovation carry on the subject "Grafos modelos $y$ aplicaciones" of the Bachelor's Degree in Informatics Engineering of the Escuela Técnica Superior de Ingeniería Informática (ETSINF) of the Universitat Politècnica de València (UPV). We comment the implementation of the methodologie and used teaching materials in the process that have lead us to it.
\end{abstract}

Keywords: competences, methodologie, evaluation, flip teaching, $O C W$, MOOC, Graph Theory.

\begin{abstract}
Resumen
En este trabajo presentamos la innovación realizada en la asignatura Grafos modelos y aplicaciones del plan de estudios del Grado en Ingeniería Informática impartida en la Escuela Técnica Superior de Ingeniería Informática (ETSINF) de la Universitat Politècnica de València (UPV). Comentamos los materiales docentes generados y utilizados, la implementación de la metodología y el proceso que nos ha llevado a ello.
\end{abstract}

Palabras clave: competencias, metodología, evaluación, flip teaching, $\mathrm{OCW}$, $M O O C$, educación inversa, teoría de grafos.

\section{Introducción}

Es un hecho irrefutable que la enseñanza universitaria ha experimentado un cambio considerable en las últimas décadas. Las causas de este cambio son múltiples, por un lado, la estabilidad y democratización de la sociedad y el desarrollo de nuevas líneas pedagógicas $y$, por otro, el crecimiento exponencial que ha experimentado la tecnología, que se ha adueñado de nuestros hogares, lugares de trabajo, etc., cambiando sustancialmente la sociedad en la que vivimos. En particular, podemos destacar las conocidas como TIC, relacionadas con internet, los medios de comunicación, generación de vídeos, etc., cuyo desarrollo ha ido acompañado de un claro abaratamiento de costes y facilidad de manejo de los diferentes dispositivos que permiten crear y editar vídeos de calidad, así como de la posibilidad de difundirlos posteriormente en la red de forma casi automática. 
Desde la experiencia que nos avala con una OCW y un MOOC premiados, presentamos en la primersa sección de este trabajo la evolución de la enseñanza de la Teoría de grafos en la Escuela de Informática (ETSINF) de la Universitat Politècnica de València (UPV), empezando en los años 90 y llegando hasta la actualidad, en la que hemos incorporado a nuestra docencia la metodología "flip teaching". Observamos que la materia mencionada ha recibido diferentes nombres y ubicaciones en los distintos cursos a lo largo de distintos planes de estudio que se han ido implantando durante este periodo.

En la segunda sección, comentamos como llevamos a cabo la metodología de la clase inversa a partir del material creado, tanto audiovisual como impreso.

\section{Evolución de la Teoría de Grafos en la ETSINF}

La Teoría de grafos empezó a impartirse en el año 1985 dentro de las materias que conformaban el plan de estudios de Ingeniero en Informática. La materia era totalmente teórica y la metodología utilizada la clase magistral, habitual en aquellos años en prácticamente todas las aulas. La participación de los alumnos en las clases era escasa. En cuanto a material docente, se disponía de los apuntes del profesor, que los alumnos compraban en repografía, los apuntes tomados en clase y las hojas de problemas. En el año 1996 publicamos un libro en la editorial Reverté (Jordán, 1996), ad hoc para la asignatura impartida en aquellos momentos, pero que si no es complementado con otros materiales, resulta muy teórico para la asignatura tal y como está concebida hoy en día. Con la aparición de los laboratorios informáticos, en los años 90 se incorporaron a la asignatura prácticas con el programa de cálculo simbólico Mathematica ${ }^{\circledR}$, al que se accedía por una red bastante inestable y deficitaria para el número de usuarios.

La asignatura cambió de nombre, y, dada la reducción de créditos que le correspondieron, se redujo su contenido, oportunidad que aprovechamos para darle un carácter mucho más aplicado.

Los autores, ante el cambio observado en la idiosincrasia de nuestros alumnos y las corrientes pedagógicas que empezaron a surgir, propugnando centrar el aprendizaje en el alumno, se plantearon modificar las metodologías docentes adecuándolas al perfil de los nuevos estudiantes. Así, aunque la principal metodología utilizada era la clase magistral, esta se tornó más y más participativa, incorporando actividades donde se pedía a los alumnos que plantearan y resolvieran semanalmente cuestiones teóricas nuevas sobre la materia que habían estudiado o propusieran problemas que se pudieran resolver previa modelización a partir de la teoría de grafos.

En 2003, la UPV decidió desarrollar Polimedia, un sistema de grabación HD de objetos de aprendizaje en vídeos de forma simple y rápida, en estudios audiovisuales que se crearon en el campus de la universidad (Turró, 2010). De esta forma, el profesorado fue animado a realizar contenidos digitales de aprendizaje de calidad, al tiempo que asesorado en cuestiones técnicas, por el programa Docencia en red que continúa actualmente en vigor en

(c)) BY-NC-ND 2017, Universitat Politècnica de València 
la UPV. Dentro de las actividades impulsadas por este programa se encuentran las Open Course Ware (OCW), iniciativa llevada a cabo inicialmente por el Instituto Tecnológico de Massachusetts en 2001 (MIT, 2017). Estas asignaturas construidas a partir sólo de texto o incluyendo vídeos, enlaces, applets, etc. constituyen el primer paso al acceso libre al conocimiento a todo aquel que disponga de internet.

Como consecuencia de estos movimientos educativos y tecnológicos, los autores crearon vídeos relativos a la teoría de grafos y empezaron a experimentar con distintas metodologías, como se refleja en los diferentes artículos publicados y ponencias presentadas al respecto (Jordán, 2008(1), 2008(2), 2009, 2010(2)).

A raíz del curso del profesor Miguel Valero (Valero 2010), impartido dentro del programa de formación del profesorado del Instituto de Ciencias de la Educación (ICE) de la UPV, la metodología de esta asignatura terminó por dar un gran salto en el curso 2010-2011. Empezamos a combinar la lección magistral con la participación de los alumnos en clase y la entrega semanal, por grupos e individualmente, de problemas que eran corregidos y devueltos la semana siguiente; acercándonos a la metodología que poco después se instauró de forma normativa, y que todos conocemos como "Bolonia".

Como consecuencia de esta nueva línea de actuación creamos una OCW para nuestra asignatura, Estructuras matemáticas para la informática 2 (Jordán, 2010(1)), que fue premiada por el OpenCourseWare Consortium en Boston, 2010. La asignatura fue y sigue siendo utilizada como material docente a la hora de impartir teoría de grafos.

Como consecuencia de la evolución de la tecnología surgieron los Massive On-line Open Courses (MOOC) (Pernías, 2012), como continuación natural de las asignaturas OCW. En principio un mismo objetivo, extender el conocimiento a todos aquellos lugares a los que llegue internet, pero en esta ocasión utilizando como base los vídeos, programando el estudio de la asignatura y evaluando el aprendizaje de los alumnos que la cursan.

Complementando la mencionada asignatura $\mathrm{OCW}$, que trata la teoría de grafos de un modo más teórico, creamos un MOOC centrado en las aplicaciones de dicha teoría: Aplicaciones de la teoría de grafos a la vida real (UPVX, (2013); EDX, (2014)(1), (2014)(2)), que recibió una mención especial en los premios MECD de la primera edición de Miriadax.

Es obligado señalar que la UPV instaló dentro de la plataforma educativa de Sakai (Sakai Project) el servicio PoliformaT que, entre otra funcionalidades, permite el intercambio de ficheros entre profesores y alumnos, correos, exámenes, etc.

En relación a las herramientas de aprendizaje, al mismo tiempo que han avanzado las tecnologías de comunicación, ha cogido impulso la metodología conocida como "flip teaching” (Bergmann, (2012), University of Washington) o clase inversa. Nuestra trayectoria nos ha llevado a implementarla en nuestras aulas a partir del material docente del que disponemos, tanto impreso como digital, y que seguimos incrementando actualmente (Jordán 2010 (3), 2011 (1), 2011 (2), 2011 (3); Cordero 2015 (1)).

En relación a este tipo de metodología, es preciso comentar que no es realmente nueva. Profesores preocupados por la docencia, que habitualmente utilizan la evaluación continua, 
la están empleando, sin necesidad de ponerle nombre. Desde nuestro punto de vista, la diferencia más importante se establece cuando se realiza a través de material audiovisual, debido al sesgo tecnológico que presentan la mayoría de nuestros alumnos.

En ocasiones nos hemos preguntado, ¿es mejor el material audiovisual que el escrito? Pensamos que no tiene porqué ser así. Pero debemos tener en cuenta que para los jóvenes de hoy el cambio tecnológico no ha existido. Cuando nacieron la tecnología estaba ya al alcance de su mano y no conciben un mundo en el que para comunicarse haya que acudir a un dispositivo fijado a una mesa o para buscar referencias sea necesario visitar una biblioteca. Asimismo, están acostumbrados a disponer de mucha información de forma rápida $\mathrm{y}$ en formatos de calidad, que a menudo simplifican las ideas que se quieren transmitir. Desde nuestro punto de vista, el utilizar únicamente este tipo de materiales conduce a que se pierde capacidad de concentración y de profundizar en los temas, dando la impresión de que se conoce una materia cuando lo único que se hace es una lectura transversal pero no un análisis en profundidad de los contenidos. Por ello, creemos que debemos proponer alguna actividad en que esta lectura rápida no sea suficiente (Cordero, $2015(2))$.

Teniendo todo esto en cuenta, impartimos la asignatura Grafos, modelos y aplicaciones utilizando educación inversa, dentro del Proyecto clase inversa de la UPV (PCI, 2016).

Además, las últimas corrientes pedagógicas hacen hincapié en que es necesario centrar la atención no sólo en los contenidos que el alumno debe adquirir sino en las competencias y habilidades que debería obtener.

En este sentido, incorporamos en la evaluación de la asignatura la creación y presentación de un póster sobre un artículo científico de actualidad en el que la teoría de grafos juegue un papel importante. Desarrollamos así las competencias transversales: Aplicación y pensamiento práctico, Conocimiento de problemas contemporáneos y Comunicación efectiva, además de aquellas naturalmente más ligadas al contenido de la asignatura como son Análisis y resolución de problemas y Comprensión e integración. Además, dado que también forma parte de la evaluación la resolución, por grupos de dos o tres alumnos, de ejercicios más complejos planteados con un mínimo de diez días de antelación, contribuimos a desarrollar la competencia Trabajo en grupo y liderazgo.

Finalmente, nos gustaría señalar que interaccionamos con otra asignatura de cuarto curso llamada Concurso de Programación, facilitando que los alumnos vean un mismo problema desde dos perspectivas distintas, ayudando por tanto a que desarrollen además la competencia transversal Pensamiento crítico.

\section{La metodología de la asignatura Grafos, modelos y aplicaciones}

La asignatura Grafos, modelos y aplicaciones (GMA) es una asignatura optativa de 4,5 créditos de cuarto, situada en el semestre B del plan de estudios del Grado en Informática impartida en la ETSINF de la UPV.

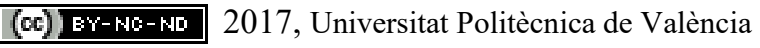


Comentamos a continuación, con más detalle, los elementos claves en la implementación de la metodología de la clase inversa en esta asignatura.

\subsection{Guía didáctica}

Como ya hemos dicho, la metodología básica utilizada es la clase inversa a partir de materiales audiovisuales. Este tipo de metodología exige, en primer lugar, una buena programación, no solo del curso, sino semana a semana. Por ello, desde el primer día de curso, en realidad una semana antes de empezar este, los alumnos van a disponer, semanalmente, de lo que llamamos guía didáctica. En ella explicitamos:

$>$ Enlaces web a la asignatura OCW y el MOOC

$>$ Vídeos a revisar antes de las clases de la semana con los enlaces respectivos

$>$ Problemas a resolver antes de la sesión de aula, donde se les preguntará sobre la solución desarrollada

$>$ Problemas a entregar, realizados por parejas, que serán debidamente corregidos y devueltos como máximo en el término de una semana

> Controles tipo test a resolver mediante la plataforma educativa PoliformaT de la UPV

$>$ Indicación aproximada de lo que se espera trabajar en cada una de las sesiones de la clase presencial

$>$ Notas, observaciones o aclaraciones sobre cualquier tema que surja en ese momento

\subsection{Preguntas flip}

Denominamos así al listado de preguntas relativas a los vídeos que los alumnos han visionado y a partir de la cual desarrollaremos una parte de la clase.

Estas preguntas, realizadas a los estudiantes por turnos, tienen un objetivo múltiple:

$>$ Permiten profundizar en la materia y favorecer el aprendizaje. Las preguntas son de dos tipos, unas en las que se revisa la materia y otras relativas a relaciones entre conceptos, cuestiones teóricas, etc. En muchas ocasiones, recogen los "puntos negros" en los que, sabemos por experiencia, los alumnos tienen tendencia a caer. Se establece un pequeño debate con "feedback" inmediato, ya sea por parte del profesor o por parte de un compañero, al respecto de si una respuesta es o no correcta, original, si hay algo que no se ha tenido en cuenta en su razonamiento, o un paso que no ha sido justificado, si hay alguna otra respuesta válida, etc.

$>$ Mejoran la expresión oral. Saber expresarse en lenguaje científico, tanto oralmente como por escrito, es necesario para un graduado en ingeniería. Por ello, verbalizar 
definiciones o razonamientos y ser corregidos al momento es de gran utilidad, ya que, además, se les da pautas para empezar la frase, formas de construir oraciones para que el contenido sea preciso y no tenga problemas de dobles interpretaciones, etc.

$>$ Fomentan la participación. Cuando un alumno no sabe la respuesta o ésta es incompleta, la pregunta rebota a otro alumno, pudiéndose establecer un pequeño debate.

$>$ La clase es más amena que si el profesor diera la misma información en una clase magistral. Al tratarse de una serie continuada de preguntas-respuestas, los alumnos están más atentos pensando en la posible respuesta, razonando si el compañero ha dado la opción correcta, etc.

$>$ Permiten conocer a los alumnos. Tras las primeras sesiones el profesor conoce los nombres de sus estudiantes, utilizándolo a la hora de dirigirse a ellos, por ejemplo en la preguntas. Todo ello genera un ambiente más cordial y en general muy agradable, que favorece el aprendizaje y además facilita la evaluación.

\subsection{Problemas puntuables}

En grupos de dos, los alumnos tienen que resolver, aproximadamente cada diez días, un boletín de problemas. Se les indica que o bien los resuelvan de forma conjunta o bien lo hagan por separado, reuniéndose después para discutir las soluciones aportadas, eligiendo por consenso la que consideren más adecuada. Es conocido que, tanto la discusión sobre un tema como la explicación de una determinada materia es una de las mejores formas de aprendizaje que existen. Además, de esta forma fomentamos el trabajo en grupo, otra de las competencias transversales que los graduados de la UPV deben obtener al finalizar sus estudios.

\subsection{Colecciones de problemas para resolver en clase.}

Una hoja de problemas por tema en los que se incluyen tanto preguntas teóricas como de aplicación a resolución de problemas reales previa modelización en teoría de grafos. Son más fáciles que los del apartado anterior; forman parte del trabajo individual, permiten conocer y asimilar la materia y sirven de preparación, junto a su posterior presentación y discusión en clase, para poder abordar los problemas puntuables comentados en el anterior apartado.

\subsection{Póster y presentación}

Al principio de curso se proporciona a los alumnos una lista de artículos científicos entre los que cada grupo, el mismo formado para la entrega de los ejercicios puntuables, debe elegir uno para su análisis, sintetización en un póster de tamaño A1 y posterior presentación ante sus compañeros y profesores. Al final de curso celebramos una mini jornada que

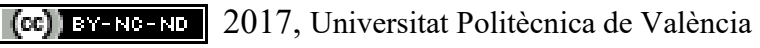


consiste en la exposición de los pósteres en el hall de la ETSINF. Tras permanecer expuestos durante una semana, los alumnos proceden a la presentación de su trabajo en un aula ante compañeros y profesores. Estos rellenarán rúbricas tanto para la presentación global como para la individual de cada ponente. Dichas rúbricas están colgadas en PoliformaT desde principio de curso, sirviendo de guía de las partes que el alumno debe trabajar cara a la realización y presentación del póster.

\subsection{Evaluación}

Creemos que la evaluación forma parte del proceso de aprendizaje, por ello consideramos los siguientes apartados:

$>$ El 20\% de la evaluación se lleva a cabo a partir de entregas de problemas. Los dos alumnos de cada grupo trabajan en equipo resolviéndolos y generando una solución conjunta

D El $20 \%$ corresponde a los controles tipo test. Se trata de exámenes tipo test en los que las preguntas se extraen aleatoriamente en cada ocasión de una batería de cuestiones. Estas cuestiones son en general de tipo teórico, relacionando conceptos. Como creemos que el objetivo de la evaluación es mejorar el aprendizaje, cada test es enviado dos veces, la de prueba, que no genera puntuación alguna y la definitiva, puntuable. De esta manera, el alumno puede darse cuenta de si conoce o no la materia antes del test que proporcionará la nota. En muchas ocasiones, al resolver el de prueba, descubre relaciones en las que no había pensado o que tenía equivocados algunos conceptos. En cualquier caso, el haber hecho la prueba no puntuable le permite profundizar en la materia, mejorar la puntuación y desde luego que su aprendizaje sea significativo

> El 25\% corresponde a la evaluación del póster y su presentación por parte del profesor mientras que el $10 \%$ es la parte de la evaluación por pares

> Finalmente, el $25 \%$ corresponde a la observación del profesor

\subsection{Relación con la asignatura Competición de programación}

La última innovación relativa a la asignatura GMA es la colaboración con la asignatura Competición de Programación (CP), que en la ETSINF responde a la necesidad que tienen los egresados de dominar la programación para superar los procesos de selección de personal en empresas de software. Detrás de las principales competiciones internacionales de programación están empresas como Google, Apple, Yahoo, Microsoft o Facebook. Y son muchas las empresas de ámbito internacional, además de las citadas, que seleccionan a sus ingenieros de software de entre los que participan en las competiciones de programación. El temario de esta asignatura cubre los aspectos más relevantes de la 
Algorítmica, donde se incluyen temas de la Teoría de grafos, aspectos que el alumno deberá utilizar y combinar adecuadamente para resolver los problemas planteados.

La asignatura GMA se plantea como uno de sus objetivos la resolución algorítmica de problemas relativos a la vida real, tipificados previa transformación en problemas teóricos en el ámbito de la teoría de grafos. Esta transformación del problema es lo que se conoce como modelización matemática. Por todo ello, aunque las asignaturas GMA y CP tengan perfiles muy diferentes, el enfoque dado a GMA resulta muy útil como apoyo a la resolución de problemas de $\mathrm{CP}$.

En este sentido, cabe recordar que uno de los objetivos principales de cualquier grado universitario es formar a los futuros egresados para que puedan seguir aprendiendo por ellos mismos a lo largo de la vida y, en buena medida, esta capacidad es la buscada por las compañías cuando valoran los resultados en competiciones de programación. Entendemos que esta experiencia es un buen ejemplo de cómo la colaboración entre profesores de distintos departamentos puede enriquecer notablemente la calidad de la enseñanza de las asignaturas implicadas al mismo tiempo que se mejora la formación de los alumnos y disminuye la dispersión de contenidos (Calvo 2016).

\subsection{Competencias transversales}

La asignatura GMA solo es punto de control, es decir, nuestra evaluación en dichas competencias aparece en el expediente de los alumnos, de las competencias transversales CT10: Conocimiento de problemas contemporáneos y CT3: Análisis y resolución de problemas. Sin embargo, como hemos comentado anteriormente, también se trabajan otras como: CT1: Comprensión e integración, CT2: Aplicación y pensamiento, CT6: Trabajo en grupo y liderazgo, CT8: Comunicación efectiva, CT9: Pensamiento crítico y CT11: Aprendizaje permanente (Alcover 2015; Jordán 2015 (1); Conejero 2015).

Todas estas competencias transversales forman parte del catálogo que la UPV ha considerado que todos sus egresados deben tener al finalizar sus titulaciones tanto de grado, como de máster.

\subsection{Valoración de la educación inversa a través de las encuestas}

A lo largo de los años hemos realizado diferentes encuestas a los alumnos.

Las primeras encuestas on-line fueron realizadas a la asignatura ya extinta, EMI2, durante el curso 2010-2011. Fue el primer año en que utilizamos la metodología de docencia inversa a partir de la OCW combinada con una evaluación continua muy controlada y el resultado fue muy bueno (Jordán 2010 (1), 2010 (2), 2011 (1), 2011 (2)). Podemos destacar los siguientes aspectos: aumentó el porcentaje de aprobados en primera convocatoria (89\%), aumentó el número de notables y sobresalientes, los alumnos opinaron que apenas habían tenido que estudiar antes del examen, pero el porcentaje de abandonos fue similar al de otro años. Sin embargo, al año siguiente repetimos la experiencia y los resultados fueron muy distintos.

La metodología inversa en la asignatura de cuarto curso GMA ha dado buenos resultados dede el primer año, 2013, en que empezó a impartirse, como consecuencia quizás del mayor

(c)) BY-NC-ND 2017, Universitat Politècnica de València 
grado de madurez de los alumnos. Estos están en general muy contentos con la metodología, piensan que el aprendizaje es muy bueno, pero al igual que todos los estudiantes, de cualquier curso y/o asignatura a los que hemos pasado una encuesta opinan que conlleva mucho más tiempo de dedicación que la tradicional (Jordán 2014 (1), 2015 (1)).

Sin embargo, la educación inversa en primer curso no aporta grandes diferencias en cuanto a resultados a los obtenidos con la metodología clásica, quizás por la mayor dificultad de que los estudiantes se involucren realmente en el proyecto. Aproximadamente el $50 \%$ de los alumnos se decantaría por esta opción (Jordán 2014 (2), 2015 (2)). Los resultados de las encuestas parecen indicar una cierta resistencia a la innovación por parte de los estudiantes. Los alumnos de la franja baja de notas prefieren una metodología tradicional, lo que en nuestra opinión se debe a que se le hace más duro tener que trabajar la materia a diario. Por su parte, el que los alumnos de la franja más alta se decanten por enseñanza tradicional puede deberse a que en esta se encuentran más cómodos y son más independientes a la hora de organizarse. Observamos que esta tendencia cambia en el cuarto curso.

\section{Conclusiones}

La metodología seguida en la asignatura GMA aúna la metodología de la clase inversa con la evaluación continua haciendo uso de la nuevas tecnologías, en lo que incluimos la asignatura OCW y MOOC, al tiempo que trabajamos diversas competencias transversales. Todo ello contribuye a mejorar el aprendizaje significativo de los contenidos trabajados en ella, pero no queremos terminar este trabajo sin dejar constancia de algunas reflexiones al respecto.

En primer lugar, como ya comentamos en la introducción, un graduado en ingeniería debe saber utilizar adecuadamente el lenguaje científico tanto oral como escrito. Los vídeos contribuyen a ello menos que los documentos escritos. Por eso, creemos conveniente reforzar este apartado recomendando estudiar parte de la materia o repasarla a partir de la parte de texto de la OCW o libros de los que recomendamos a principio de curso en la bibliografía, además de la práctica que obtenemos con el trabajo continuado en clase.

En segundo lugar, el éxito de esta metodología, y en realidad de cualquier otra, depende de la actitud de profesor y alumno. Pero la "flip teaching" exige al estudiante que se involucre más, que mantenga una actitud más activa en clase. Las técnicas que utilizamos fomentan, en general, la motivación, pero requieren la responsabilidad, por parte del alumno, de un trabajo continuado al que, aunque a medio-largo plazo da mejores resultados, en muchas ocasiones no está acostumbrado y no siempre está dispuesto a realizar. Por todo ello, el éxito de nuestra metodología depende mucho más de esta actitud que otras metodologías más tradicionales a las que el alumno está habituado, como la lección magistral. 


\section{Referencias}

ALCOVER et al., (2015) "Influencia de la educación inversa en el aprendizaje y adquisición de competencias transversales" En: Congreso Nacional de Innovación Educativa y Docencia en Red = Congrés Nacional d'Innovació Educativa i Docència en Xarxa (IN-RED 2015)

BERGMANN, J. AND SAMS, A., (2012) "Flip Your Classroom: Talk to Every Student in Every Class Every Day". En: International Society for Technology in Education

CALVO et al. (2016) "Modelos de la teoría de grafos aplicados a problemas de competiciones de programación" En: Congreso Nacional de Innovación Educativa y Docencia en Red (IN-RED 2016)

CONEJERO, J.A. y JORDÁN, C., (2015) "El póster científico como medio para desarrollar la competencia de comunicación." En: XIII Jornadas de Redes de Investigación en Docencia Universitaria. Nuevas estrategias organizativas y metodológicas en la formación universitaria para responder a la necesidad de adaptación y cambio

CORDERO, A., JORDÁN, C., TORREGROSA, J. R., (2015) "How do current students face math problems solving?" En: Proceedings of 9th International Technology, Education and development Conference, INTED, pp. 4716-4721, Madrid, 2015.

CORDERO, A., JORDÁN, C., TORREGROSA, J. R., SANABRIA-CODESAL, E., (2015), "Towards a better learning model through OCWs and MOOCs". International Journal of artificial intelligence and interactive multimedia, vol 3, No. 4, pp. 26-30, 2015.

EDX: APLICACIONES DE LA TEORÍA DE GRAFOS A LA VIDA REAL (I) .

https://www.edx.org/course/aplicaciones-de-la-teoria-de-grafos-la-upvalenciax-tgv201x-1>[Consulta: 2 de abril de 2017][ Cristina Jordán y Alberto Conejero]

EDX: APLICACIONES DE LA TEORÍA DE GRAFOS A LA VIDA REAL (II) en edX. https://www.edx.org/course/aplicaciones-de-la-teoria-de-grafos-la-upvalenciax-tgv201x-2$0>$ [Consulta: 2 de abril de 2017][ Cristina Jordán y Alberto Conejero]

JORDÁN LLUCH, C. (2015) "Desarrollo de competencias transversales en el aula y Experiencias Flipped Classroom", En: Congreso Nacional de Innovación Educativa y Docencia en Red = Congrés Nacional d'Innovació Educativa i Docència en Xarxa (IN-RED 2015)

JORDÁN LLUCH, C. y TORREGROSA SÁNCHEZ, J.R., (1996). Introducción a la teoría de grafos y sus algoritmos. Valencia: Editorial Reverté. [dos/tres autores: todos]

JORDÁN LLUCH, C. y TORREGROSA SÁNCHEZ, J.R., (2008) "Docencia en red: un paso adelante en el uso de nuevas tecnologías", En: Proc. de las VI Jornadas de redes de investigación en docencia universitaria, (2008. Alicante) p.726.

JORDÁN LLUCH, C. y TORREGROSA SÁNCHEZ, J.R., (2008), "Los objetos de aprendizaje y el nuevo contexto educativo". En: Actas del V Congreso Iberoamericano de Docencia Universitaria, (2008, Valencia).

JORDÁN LLUCH, C. (2009), "Utilización correcta e incorrecta de los ficheros Polimedia" En: Actas de la JIDINF'09 (2009, Valencia).

JORDÁN, C., "Estructuras Matemáticas para la Informática II “[On line]. Available: http://www.upv.es/ocwasi/2010/6024, [Cristina Jordán, 2010].

(cc) EY-NC-ND 2017, Universitat Politècnica de València 
JORDÁN LLUCH, C. y TORREGROSA SÁNCHEZ, J.R., (2010). "Las OCW en el nuevo contexto educativo", En: Actas de las VIII Jornadas de Redes de Investigación en Docencia Universitaria, (2010, Alicante)

JORDÁN, C., (2010), “Adaptación de la asignatura EMI2 al nuevo contexto educativo", En: Actas de la JIDINF'10, 2010, Valencia.

JORDÁN, C., SANABRIA-CODESAL, E., (2011), "La asignatura OCW Estructuras matemáticas para la informática II y los créditos ECTS”, En: XIX CUIEET, Barcelona. 2011.

JORDÁN, C., (2011), "Evaluación continua en la asignatura Estructuras matemáticas para la informática II", En: IX Jornadas de Redes de investigación en docencia Universitaria, pp. 26-36, 2011, Alicante.

JORDÁN, C., (2011),’Una experiencia de evaluación continua”, En: Actas de la JIDINF’11, 2011, Valencia.

JORDÁN, C., PÉREZ, M.J., SANABRIA-CODESAL, E., (2014), “"Flipped clasroom”: Reflexiones y opiniones de los implicados”, En: Congreso Nacional de Innovación Educativa y Docencia en Red, INRED, 2014.

JORDÁN, C., PÉREZ, M.J., SANABRIA-CODESAL, E., (2014), "Investigación del impacto en un aula de matemáticas al utilizar "flip education"”, En: Pensamiento Matemático, Vol. 4. No. 2, pp.922,2014

JORDÁN, C., PÉREZ, M.J., SANABRIA-CODESAL, E., (2015), "Educación inversa, una metodología innovadora ¿Coincide la percepción que tienen los alumnos de ella con la nuestra?" En: XIII Jornadas de Redes de Investigación en Docencia Universitaria. Nuevas estrategias organizativas y metodológicas en la formación universitaria para responder a la necesidad de adaptación y cambio, Alicante, 2015

JORDÁN, C., SANABRIA-CODESAL, E., (2015), ¿Qué opinan de la metodología "flip teaching" loa alumnos de nuevo ingreso?"XXIII Congreso universitario de innovación educativa, 2015.

MIT OPENCURSEWARW/FREE ONLINE COURSE MATERIALS-MASSACHUSSETTS (2017)

$<$ https: \locw.mit.edu>

OPEN EDUCATION CONSORTIUM < http://www.oeconsortium.org>

PerníAS, P. y LuJÁn-MorA, S., (2012), "MOOC. Los MOOC: Orígenes, historia y tipos" En : Comunicación y Pedagogía 269-270. <http://www.centrocp.com/los-mooc-origeneshistoria-y-tipos>

PROYECTO CLASE INVERSA (PCI), (2017), Universitat Politécnica de Valencia. $<$ http://claseinversa.blogs.upv.es/el-proyecto/proyecto-clase-inversa-upv/> [Consulta: 2 de abril de 2017]

Turro, C., CAÑERo, A., Busquets, J., (2010). "Video Learning Objects Creation with Polimedia”. En: IEEE International Symposium on Multimedia, pp. 371-376.

\section{SAKAI PROJECT https://sakaiproject.org/}

UNIVERSITAT POLITĖCNICA DE VALÈNCIA, (2017). Proyecto institucional Incorporación de las competencias transversales en el currículo de los egresados de la UPV.

$<$ http://www.upv.es/entidades/ICE/info/U0724624.pdf> [Consulta: 31 de marzo de 2017] 
UNIVERSITY OF WASHINGTON. FLIPPING THE CLASSROOM

$<$ http://www.washington.edu/teaching/teaching-resources/engaging-students-in-

learning/flipping-the-classroom/>[Consulta: 29 de abril de 2015]

UPVX. Aplicaciones de la Teoría de Grafos a la vida real

$<$ http://cursografos.upvx.es/ficha $>$ [Consulta: 2 de abril de 2017][ Cristina Jordán y Alberto Conejero]

VALERO-GARCÍA, M. ESPONA, M., (2010), "Adaptación de asignaturas al EEES".

Curso de formación permanente del ICE de la UPV, Valencia, 2010. 